\section{Spontaneous closure of dural arterio-venous fistula}

Sir,

Dural arteriovenous fistulas (DAVFs) account for $10-15 \%$ of the intracranial arteriovenous malformations. Though a subgroup of DAVFs has been reported to have a benign course complete spontaneous disappearance of DAVFs is uncommon.

A 25-year-old male presented to us with history of headache and pulsatile tinnitus for three months with no deficits on neurological examination. Digital subtraction angiography (DSA) showed a Borden Type II DAVF with no venous ectasia [Figure 1]. In view of the symptomatic nature of the lesion with Type II DAVF, the patient was planned for embolization by a transarterial route. He was, however, lost to follow-up and came back after one year of diagnosis with resolution of tinnitus and presence of mild headache. A repeat DSA performed before undertaking any definitive procedure revealed complete disappearance of the DAVF [Figure 2]. The patient was doing well at last follow-up with Magnetic resonance imaging/Magnetic resonance angiography showing no evidence of any vascular malformation.

Dural arteriovenous fistulas have their arterial supply from perforating branches of extracranial arteries or from branches of meningeal arteries with drainage to dural venous sinuses or leptomeningeal veins. Natural history of DAVFs is variable and depends on location and venous drainage characteristics..$^{[1,2]}$ Though a significant number of transverse-sigmoid sinus DAVFs follow a benign course, total spontaneous occlusion as described in our case of symptomatic Type II DAVF is very rare. ${ }^{[1-4]}$ Sinus thrombosis and intracranial hemorrhage have been proposed in cases of a few DAVFs which thrombosed spontaneously, none of which was present in the case described. ${ }^{[4]}$ We believe that this spontaneous closure could be explained by the intrinsic compression of the arteriovenous shunts within the sinus wall. A focal increase in sinus size could lead to focal compression of the arteriovenous shunts within the wall itself, thus leading to the final occlusion of the DAVF. Secondary vasospasm leading to apparent non-visualization of the DAVF on DSA was ruled by MRI performed one year later which did not reveal any DAVF confirming total spontaneous closure. Though benign natural history is the rule in most of the lateralsigmoid DAVFs, spontaneous closures even in these cases is not very common. The presently reported case of spontaneous closure of a Type II DAVF represents evidence of good outcome in a DAVF in the absence of any treatment throwing further insight into the natural history of these lesions that can have prognostic and therapeutic implications.

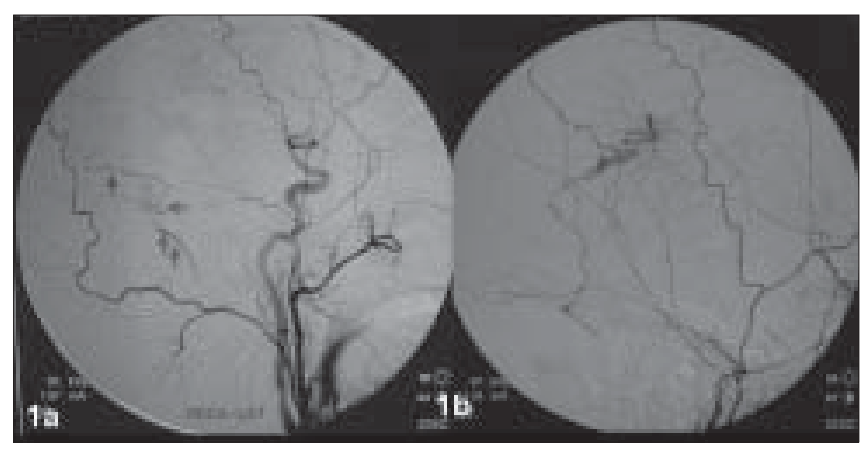

Figure 1: Digital Subtraction Angiography (DSA) showing a DAVF supplied by the branches of the right middle meningeal artery and draining into subarachnoid veins and the transverse sinus with no evidence of any venous ectasia



Figure 2: Repeat DSA showing no evidence of any dural arteriovenous fistula

\section{Noufal Basheer, Manish K. Kasliwal, Shailesh Gaikwad ${ }^{1}$, Bhawani S. Sharma}

Departments of Neurosurgery and ${ }^{1}$ Neuroradiology, Neurosciences Centre, All India Institute of Medical Sciences, New Delhi - 110029 , India.E-mail: drsharmabs@yahoo.com

\section{References}

1. Luciani A, Houdart E, Mounayer C, Saint Maurice JP, Merland JJ. Spontaneous closure of dural arteriovenous fistulas: Report of three cases and review of the literature. Am J Neuroradiol 2001;22:992-6.

2. Pritz MB, Pribram HF. Spontaneous closure of high risk dural arteriovenous malformations of transverse sinus. Surg Neurol 1991;61:226-8.

3. Hansen JH, Sogaard I. Spontaneous regression of an extra and intracranial arteriovenous malformation: Case report. J Neurosurg 1976;45:338-41.

4. Kutluk K, Schumacher M, Mironov A. The role of sinus thrombosis in occipital dural arteriovenous malformations-development and spontaneous closure. Neurochirurgia 1991;34:144-7.

Accepted on 09-05-2008 